\title{
Anti-Angiogenics: Current Situation and Future Perspectives
}

\author{
Katja Zirlik ${ }^{a, b}$ Justus Duyster ${ }^{a}$ \\ a University Medical Center Freiburg, Department of Medicine I: Hematology, Oncology, and Stem-Cell Transplantation, \\ Freiburg i.Br., Germany,

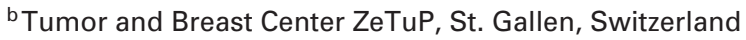

\section{Keywords}

Anti-angiogenics · Angiogenesis - VEGF .

Monoclonal antibodies - Tyrosine kinase inhibitors

\section{Summary}

Angiogenesis, the process leading to the formation of new blood vessels, is one of the hallmarks of cancer. Extensive studies established that i) vascular endothelial growth factor (VEGF) is a key driver of sprouting angiogenesis, ii) VEGF is overexpressed in most solid cancers, and iii) inhibition of VEGF can suppress tumor growth in animal models. This has led to the development of pharmacological agents for anti-angiogenesis to disrupt the vascular supply and starve the tumor of nutrients and oxygen, primarily through the blockade of VEGF/VEGF receptor signaling. This effort has resulted in 11 antiVEGF drugs approved for certain advanced cancers, either alone or in combination with chemotherapy and other targeted therapies. However, inhibition of VEGF signaling is not effective in all cancers, and anti-angiogenics have often only limited impact on overall survival of cancer patients. This review focuses on the current status of FDA-approved anti-angiogenic antibodies and tyrosine kinase inhibitors and summarizes the progress and future directions of VEGF-targeted therapy.

\section{Introduction}

Angiogenesis, the growth of new blood vessels from pre-existing blood vessels via a process called sprouting, is one of the hallmarks of cancer. Angiogenesis is a multistage process regulated by numerous growth factors and their receptors. Targeting angiogenesis as a tumor therapy was first hypothesized over 4 decades ago by Judah Folkman [1]. Of all the identified molecules leading to blood vessel formation, vascular endothelial growth factor A (VEGF-A) appears to be the main molecular driver of tumor angiogenesis. Indeed, VEGF-A is overexpressed in the majority of solid tumors and for this reason is the dominant target for anti-angiogenic drugs [2]. The main signaling tyrosine kinase receptor is VEGF receptor 2 (VEGFR2); 2 other VEGFRs include VEGFR1 (also known as FLT1) and VEGFR3 (also known as FLT3).

Three approaches have been developed for targeting the VEGF signaling pathway:

Ligand binding agents that block the binding of VEGF ligands to receptors (e.g., bevacizumab which binds to VEGF-A alone and aflibercept which binds to VEGF-A, VEGF-B, and placental growth factor (PLGF)); antibodies that block signaling though VEGFRs (e.g., ramucirumab which binds to VEGFR2); and tyrosine kinase inhibitors (TKIs) which block the kinase activity of VEGFR1, VEGFR2, and VEGFR3 (e.g., sorafenib, sunitinib, pazopanib) (table 1). TKIs often inhibit the kinase activity of some other receptor tyrosine kinases such as platelet-derived growth factor receptors (PDGFRs), c-KIT, and FLT3.

\section{Current Situation}

\section{Antibody-Based Therapies}

VEGF is overexpressed in a vast majority of solid tumors and is widely considered to be a key player in mediating tumor angiogenesis $[2,3]$. For this reason, over the past decades, the development of anti-angiogenics predominantly focused on the development of VEGF/VEGFR inhibitors. Preclinical evidence showed that monotherapy of VEGF blockade inhibited tumor growth in subcutane-

\section{KARGER}

(c) 2018 S. Karger GmbH, Freiburg 
Table 1. FDA-approved vascular endothelial growth factor (VEGF)-targeted therapy for oncology

\begin{tabular}{|c|c|c|c|}
\hline Mechanism & Drug & Brand name & Indications \\
\hline Monoclonal anti-VEGF antibody (VEGF-A) & bevacizumab & Avastin & $\begin{array}{l}\text { mCRC, NSCLC, GBM, RCC, epithelial ovarian cancer, } \\
\text { fallopian tube cancer, primary peritoneal cancer, } \\
\text { cervical cancer }\end{array}$ \\
\hline Recombinant fusion VEGF protein (VEGF-A, VEGF-B, PLGF) & aflibercept & Zaltrap & $\mathrm{mCRC}$ \\
\hline Monoclonal anti-VEGFR2 antibody & ramucirumab & Cyramza & $\begin{array}{l}\text { mCRC, NSCLC, gastric or gastroesophageal junction } \\
\text { adenocarcinoma }\end{array}$ \\
\hline Multi-TKI (VEGFRs, PDGFRs, RAF, KIT, FLT3, RET) & sorafenib & Nexavar & RCC, HCC, thyroid cancer \\
\hline Multi-TKI (VEGFRs, PDGFRs, FLT3, CSF1R, RET) & sunitinib & Sutent & $\begin{array}{l}\text { RCC, pancreatic neuroendocrine tumors, } \\
\text { gastrointestinal stromal tumors }\end{array}$ \\
\hline Multi-TKI (VEGFRs, PDGFRs, FGFRs, TIE2, KIT, RET, RAF) & regorafenib & Stivarga & GIST, mCRC, HCC \\
\hline Multi-TKI (VEGFRs, PDGFRs, FGFR1-2, c-Kit) & pazopanib & Votrient & RCC, soft tissue sarcoma \\
\hline Multi-TKI (VEGFRs, PDGFRs, c-Kit) & axitinib & Inlyta & RCC \\
\hline Multi-TKI (VEGFRs, EGFR, RET) & vandetanib & Caprelsa & medullary thyroid cancer \\
\hline Multi-TKI (VEGFRs, Tie2, cMet, AXL) & cabozantinib & Cometriq & medullary thyroid cancer, RCC \\
\hline
\end{tabular}

TKI = Tyrosine kinase inhibitor; $\mathrm{mCRC}=$ metastatic colorectal cancer; NSCLC = non-small cell lung cancer; GBM = glioblastoma multiforme; RCC = renal cell carcinoma; HCC = hepatocellular carcinoma; GIST = gastrointestinal stromal tumor.

ous human xenografts of many cancer types. Anti-VEGF treatment even resulted in a marked decrease in metastasis, in particular in preclinical colorectal cancer models [4].

\section{Bevacizumab}

Ferrara et al. [5, 6] designed and developed the first anti-angiogenic inhibitor, bevacizumab, a recombinant humanized monoclonal antibody (mAb) blocking VEGF-A. Intravenous administration of bevacizumab depletes VEGF in the bloodstream and in perfused tissues, inhibiting the interaction between VEGF and VEGFRs [3]. Several clinical trials of bevacizumab with chemotherapy showed increased overall survival (OS) and/or progression-free survival (PFS) in metastatic colorectal cancer (mCRC) (in first and second line) [7-9], as well as in cervical cancers, non-small cell lung cancer (NSCLC), ovarian cancer, and mesothelioma [10-14]. A combination of bevacizumab and interferon alpha immunotherapy is now one of the standards of care in metastatic renal cell carcinoma (mRCC) prolonging PFS [15].

To date, bevacizumab is Food and Drug Administration (FDA)approved for the treatment of CRC, NSCLC, glioblastoma, RCC, cervical cancer, ovarian cancer, fallopian tube cancer, and peritoneal cancer (table 1). However, in the majority of cancers, bevacizumab failed to increase survival, including breast, melanoma, pancreatic, and prostate cancer [16]. The precise explanation as to why anti-angiogenic agents show efficacy in some metastatic cancers and not others, is currently unknown. Conceivably, important differences in the vascular biology of these cancers may underlie the discrepant results seen with this approach across different tumors.

Of note, the history of anti-angiogenic therapy in the treatment of metastatic breast cancer is of significant interest. Bevacizumab was FDA-approved in combination with paclitaxel for the treatment of HER2-negative metastatic breast cancer in February 2008 under the FDA's accelerated approval program. 3 further phase III trials of bevacizumab in combination with chemotherapy in HER2- negative metastatic breast cancer (AVADO, RIBBON-1, and RIBBON-2) demonstrated an extension of PFS, but no effect on OS, when compared to chemotherapy alone [17-19]. As a consequence of these results, the FDA withdrew its approval for bevacizumab in this indication in November 2011.

\section{Ramucirumab}

Recently, 2 phase III studies evaluated the role of ramucirumab, a VEGFR2 mAb interfering with VEGFs binding to their receptor. The REGARD study evaluated ramucirumab as second-line therapy after disease progression on a first-line chemotherapy regimen in patients with advanced, unresectable gastroesophageal tumors [20]. Median OS was 5.2 months in the ramucirumab group and 3.8 months in the placebo group ( $\mathrm{p}=0.047)$. A longer PFS $(2.1$ months for ramucirumab vs. 1.3 months for placebo) was also reported. Overall, this study identified ramucirumab as the first biological treatment given as a single drug showing survival benefits in patients with advanced gastroesophageal adenocarcinomas progressing after first-line chemotherapy. A second phase III study (RAINBOW) investigated ramucirumab combined with paclitaxel, as second-line treatment in patients with metastatic gastric cancer who progressed after a first-line chemotherapy [21]. OS was significantly longer in the ramucirumab plus paclitaxel group than in the placebo group (9.6 vs. 7.4 months). Furthermore, ramucirumab plus paclitaxel significantly delayed disease progression (PFS 4.4 vs. 2.9 months) and increased the response rate (28 vs. $16 \%$ ). Based on these results, ramucirumab was approved by the FDA and the European Commission either as a single agent or in association with paclitaxel in patients with advanced or metastatic gastric and gastroesophageal junction cancer after progression on fluoropyrimidine or platinum-containing regimens. Ramucirumab also showed increased OS when combined with docetaxel for second-line treatment of metastatic NSCLC [22] and mCRC [23], leading to FDA approval also in these indications. 


\section{Aflibercept}

Aflibercept is a novel fusion protein that binds to 3 VEGF family ligands: VEGF-A, VEGF-B, and PLGF. Aflibercept combined with chemotherapy as second-line treatment in mCRC patients showed a small OS (13.5 months in the aflibercept arm vs. 12 months in the control arm; $\mathrm{p}=0.0032)$ and PFS (6.9 months in the aflibercept arm vs. 4.7 months in the control arm; $\mathrm{p}<0.0001$ ) benefit in a randomized phase II clinical trial [24]. Based on these data, aflibercept was recently approved for the treatment of mCRC when given in combination with chemotherapy. Furthermore, experimental models propose aflibercept as a promising candidate to treat hepatocellular carcinoma (HCC) [25], a highly vascular tumor with the development of neoarteries in parallel with tumor growth.

\section{Small Molecule Inhibitors}

In addition to monoclonal antibodies, TKIs were developed to inhibit VEGFRs and their downstream targets in order to suppress endothelial proliferation and disrupt the vascular supply of nutrients and oxygen. Multiple TKIs have been approved as single therapies in specific indications based on improvement of OS or PFS in phase III trials. These include sorafenib, sunitinib, axitinib, regorafenib, pazopanib, vandetanib, cabozantinib, and lenvatinib (table 1).

Among this class of agents, the pioneer drugs were sorafenib and sunitinib. Subsequently, other agents emerged with similar modes of action but better toxicity profiles. This second-generation of multi-kinase inhibitors shows improved target affinity combined with less off-target effects.

Four of these FDA-approved agents, namely sorafenib, sunitinib, pazopanib, and axitinib, proved to be highly successful in mRCC and are FDA-approved in this setting. A phase III trial comparing sunitinib with pazopanib demonstrated that both drugs have similar efficacy [26], and single-agent therapy with either drug is now recommended as standard of care in the first line in mRCC. TKIs also showed single-agent activity in advanced HCC (sorafenib) and advanced pancreatic neuroendocrine tumors (PNET) (sunitinib). Regarding mCRC, despite the benefit observed when bevacizumab or aflibercept are combined with chemotherapy, efforts to combine anti-angiogenic TKIs with chemotherapy have so far generated disappointing results in terms of improving OS $[27,28]$. However, single-agent treatment with the TKI regorafenib was recently demonstrated to moderately extend OS compared to placebo (6.4 vs. 5.0 months; $\mathrm{p}=0.0052)$ and PFS (1.9 vs. 1.7 months; $\mathrm{p}<0.0001)$ in mCRC patients who had previously progressed on standard therapies [29]. Regorafenib is now approved for the treatment of mCRC in this setting in Europe and the United States. Notably, in March 2016, Bayer pulled regorafenib back on the German market after the Federal Joint Committee (GBA) decided that regorafenib has no added value for the treatment of CRC and that its disadvantages outweigh its benefits. The decision of the G-BA was described by the company as incomprehen- sible', especially since such additional benefits of regorafenib had been previously recognized. The European approval, however, was not affected, and regorafenib is still available in other European countries. Regorafenib recently also obtained approval for the additional indication of gastrointestinal stromal tumors (GIST) and HCC. Regorafenib is the first and only treatment that has demonstrated a significant improvement in OS in second-line HCC patients [30].

Among the second-generation multi-kinase inhibitors, also pazopanib, cabozantinib, lenvatinib, axitinib, and vandetanib have been approved as monotherapies in specific indications (table 1).

Recently, based on the results of the phase III LUME-Lung 1 trial [31], the European Medicines Agency (EMA), but not FDA, approved the use of nintedanib, an oral multi-kinase inhibitor targeting VEGFR1-3, FRGFR1-3, PDGFRa-b, RET, FLT3, and Src family kinases, combined with docetaxel for the second-line treatment of NSCLC.

Cediranib is a pan-VEGFR inhibitor showing prolonged PFS when combined with platinum-based chemotherapy in relapsed platinum-sensitive ovarian cancer [32]. The OS endpoint is currently being analyzed for this clinical trial; however, the trial met the endpoint and therefore the drug will likely be approved by the FDA.

Among the antiangiogenic agents under investigation in gastric cancer is apatinib, an oral VEGFR2 inhibitor. In a phase III trial, patients progressing on second-line therapy were randomized to apatinib or placebo. Median OS (4.7 months with placebo vs. 6.5 months with apatinib) and PFS (1.8 vs. 2.6 months) were significantly improved [33].

Anti-angiogenic regimens targeting the excess of angiogenic inducers including bevacizumab or aflibercept show clinical benefits when associated with cytotoxic therapies, as reported above. In contrast, TKIs do not show any clinical improvement when administered with standard therapies. Attempts to combine anti-angiogenic TKIs with chemotherapy did not improve PFS in mCRC and metastatic breast cancer. Indeed, VEGFR TKIs exhibit single-agent activity and are effective as monotherapy while showing toxicity in combination with chemotherapy [34].

\section{Future Perspectives}

The overall benefits of anti-angiogenics from the perspective of impacting survival have left much to be desired, endorsing the need for developing more effective therapeutic regimens.

Especially, immune checkpoint inhibition has now been clinically validated as an effective treatment for various tumors with promising results and there is exceptional potential for combining immunotherapy agents with conventional cancer treatments.

The programmed death protein 1 (PD-1), its ligand the programmed death ligand 1 (PD-L1), and the cytotoxic T-lymphocyteassociated antigen 4 (CTLA-4) are negative regulators of T-cell immune function. Direct stimulation of the immune system with antibodies against PD-1, PD-L1, and CTLA-4 leads to tremendous 
clinical efficacy in multiple cancers, having resulted in the FDA approval of the following immune checkpoint targeted immunotherapies: ipilimumab $\left(\right.$ Yervoy $^{\circledR}$, Bristol-Myers Squibb GmbH \& Co. KGaA, Munich, Germany), pembrolizumab (Keytruda ${ }^{\circledR}$, MSD Sharp \& Dohme GmbH, Munich, Germany), nivolumab (Opdivo ${ }^{\circledR}$, Bristol-Myers Squibb), atezolizumab (Tecentriq ${ }^{\circledR}$, Roche Pharma AG, Grenzach-Whylen, Germany), avelumab (Bavencio ${ }^{\circledR}$, Merck / Pfizer Pharma GmbH, Berlin, Germany), and durvalumab (Imfinzi ${ }^{\circledR}$, AstraZeneca, Wedel, Germany).

Notably, VEGF was recognized as one of the critical molecules of immunosuppression. VEGF suppresses dendritic cell differentiation and activity [11] and expands T regulatory cells and myeloidderived suppressor cells. In addition, in patients with CRC, VEGF inhibition by bevacizumab improved the antigen-presenting capacity of circulating dendritic cells [35], revealing an additional mechanism for bevacizumab on immune functions in the context of checkpoint blockade. The positive effect on immune response obtained by inhibiting the VEGF pathway can be further increased by combined blockade of angiopoietin-2 (Ang-2) [36, 37]. Besides VEGF, Ang-2 is an important player in angiogenesis. A bispecific antibody which binds both VEGF-A and Ang-2 showed a better effect as compared to single blockade in many preclinical models and synergized with PD-1 blockade [36, 38].

More recently, a preclinical study provided evidence that antiPD-1 or anti-PD-L1 therapy sensitized and prolonged the efficacy of antiangiogenic therapy, and conversely, antiangiogenic therapy improved anti-PD-L1 treatment by supporting vascular changes such as high endothelial venule formation and vessel normalization that facilitate enhanced cytotoxic T-cell infiltration and subsequent tumor cell destruction [39]. In addition, Tian et al. [40] also proposed that CD4+ T-lymphocyte activation by immune checkpoint blockade increased vessel normalization, as indicated by increased pericyte coverage, improved tumor vessel perfusion, and reduced vascular permeability, resulting in altered tumor progression.

Based on these preclinical and translational data supporting synergy between anti-angiogenics and immunotherapy, multiple trials of combining VEGF-targeted therapy and checkpoint inhibitors are underway. Clinical trials in many different indications such as melanoma, CRC, RCC, NSCLC, and glioblastoma are evaluating e.g., i) bevacizumab in combination with ipilimumab, atezolizumab, nivolumab, or pembrolizumab, ii) aflibercept in combination with pembrolizumab, or iii) the TKIs sunitinib, axitinib, or cabozantinib in combination with nivolumab, pembrolizumab, or avelumab (clinicaltrials.gov).
Recently, a clinical study of a combination therapy using the anti-CTLA4 antibody ipilimumab with the anti-VEGF antibody bevacizumab reported promising efficacy in patients with advanced melanoma resulting in a median OS of more than 2 years [41]. High-grade toxicity was more common than expected for either drug alone, but was manageable. Interestingly, the combination led to an accumulation of $\mathrm{CD} 8^{+} \mathrm{T}$ cells and dendritic cells in the tumor microenvironment - suggesting synergism of immunotherapeutic effector mechanisms and warranting further investigation of this combination.

Furthermore, nanotechnology-based approaches could improve the current pharmacokinetic profiles of anti-angiogenic drugs and favor their selective accumulation in tumors [42]. Recently, the humanized tri-specific nanobody BI 836880 comprising 2 single-variable domains blocking VEGF and Ang2, and an additional albumin module for half-life extension in vivo was generated. This novel VEGF/Ang2-blocking nanobody showed promising properties in vitro and in vivo which strongly support the evaluation of this molecule in the clinic. At present, 2 phase I dose escalating studies are underway in patients with advanced solid tumors exploiting this nanobody (NCT02689505, NCT02674152).

\section{Conclusion}

Clinical development of anti-angiogenic therapy was a success and translated into increased OS and PFS in many tumors. This is in line with the concept that tumor angiogenesis is a hallmark of cancer. However, the benefits are limited, and predictive biomarkers for this class of agent remain elusive. One explanation for the disappointing results is the existence of intrinsic or acquired resistance to anti-angiogenic therapy mediated by both tumor cells and stromal cells. Given the recent success of immunotherapies, and since VEGF promotes an immunosuppressive tumor microenvironment, combinations of anti-angiogenics with checkpoint blockers have become an attractive strategy. Other emerging directions for medical treatment targeting angiogenesis are bispecific antibodies binding VEGF and Ang-2 and nanotechnology approaches. In addition, understanding the mechanism through which stromal cells mediate resistance in the tumor would help to improve the efficacy and durability of anti-angiogenic therapy.

\section{Disclosure Statement}

The authors have declared that no competing interests exist.

\section{References}

1 Folkman J: Tumor angiogenesis: therapeutic implications. N Engl J Med 1971;285:1182-1186.

2 Ferrara N: VEGF and the quest for tumour angiogenesis factors. Nat Rev Cancer 2002;2:795-803.

3 Ferrara N, Gerber HP, LeCouter J: The biology of VEGF and its receptors. Nat Med 2003;9:669-676.
4 Ferrara N, Carver-Moore K, Chen H, Dowd M, Lu L, O'Shea KS, Powell-Braxton L, Hillan KJ, Moore MW: Heterozygous embryonic lethality induced by targeted inactivation of the VEGF gene. Nature 1996;380:439442
5 Ferrara N, Hillan KJ, Novotny W: Bevacizumab (Avastin), a humanized anti-VEGF monoclonal antibody for cancer therapy. Biochem Biophys Res Commun 2005; 333:328-335.

6 Ferrara N, Kerbel RS: Angiogenesis as a therapeutic target. Nature 2005;438:967-974. 
7 Giantonio BJ, Catalano PJ, Meropol NJ, O’Dwyer PJ Mitchell EP, Alberts SR, Schwartz MA, Benson AB 3rd: Bevacizumab in combination with oxaliplatin, fluorouracil, and leucovorin (FOLFOX4) for previously treated metastatic colorectal cancer: results from the Eastern Cooperative Oncology Group Study E3200. J Clin Oncol 2007;25:1539-1544.

8 Hurwitz H, Fehrenbacher L, Novotny W, Cartwright T, Hainsworth J, Heim W, Berlin J, Baron A, Griffing S, Holmgren E, Ferrara N, Fyfe G, Rogers B, Ross R, Kabbinavar F: Bevacizumab plus irinotecan, fluorouracil, and leucovorin for metastatic colorectal cancer. N Engl J Med 2004;350:2335-2342.

9 Saltz LB, Clarke S, Diaz-Rubio E, Scheithauer W, Figer A, Wong R, Koski S, Lichinitser M, Yang TS, Rivera F, Couture F, Sirzen F, Cassidy J: Bevacizumab in combination with oxaliplatin-based chemotherapy as first-line therapy in metastatic colorectal cancer: a randomized phase iii study. J Clin Oncol 2008;26:2013-2019.

10 Aghajanian C, Blank SV, Goff BA, Judson PL, Teneriello MG, Husain A, Sovak MA, Yi J, Nycum LR: OCEANS: a randomized, double-blind, placebo-controlled phase iii trial of chemotherapy with or without bevacizumab in patients with platinum-sensitive recurrent epithelial ovarian, primary peritoneal, or fallopian tube cancer. J Clin Oncol 2012;30:2039-2045.

11 Gabrilovich D, Ishida T, Oyama T, Ran S, Kravtsov V, Nadaf S, Carbone DP: Vascular endothelial growth factor inhibits the development of dendritic cells and dramatically affects the differentiation of multiple hematopoietic lineages in vivo. Blood 1998;92:4150-4166.

12 Perren TJ, Swart AM, Pfisterer J, Ledermann JA, PujadeLauraine E, Kristensen G, Carey MS, Beale P, Cervantes A, Kurzeder C, du Bois A, Sehouli J, Kimmig R, Stahle A, Collinson F, Essapen S, Gourley C, Lortholary A, Selle F, Mirza MR, Leminen A, Plante M, Stark D, Qian W, Parmar MK, Oza AM: A phase 3 trial of bevacizumab in ovarian cancer. N Engl J Med 2011;365:2484-2496.

13 Tewari KS, Sill MW, Long HJ 3rd, Penson RT, Huang H, Ramondetta LM, Landrum LM, Oaknin A, Reid TJ, Leitao MM, Michael HE, Monk BJ: Improved survival with bevacizumab in advanced cervical cancer. $\mathrm{N}$ Engl J Med 2014;370:734-743.

14 Zalcman G, Mazieres J, Margery J, Greillier L, Audigier-Valette C, Moro-Sibilot D, Molinier O, Corre R, Monnet I, Gounant V, Riviere F, Janicot H, Gervais R, Locher C, Milleron B, Tran Q, Lebitasy MP, Morin F, Creveuil C, Parienti JJ, Scherpereel A: Bevacizumab for newly diagnosed pleural mesothelioma in the Mesothelioma Avastin Cisplatin Pemetrexed Study (MAPS) a randomised, controlled, open-label, phase 3 trial. Lancet 2016;387:1405-1414.

15 Rini BI, Bellmunt J, Clancy J, Wang K, Niethammer AG, Hariharan S, Escudier B: Randomized phase iii trial of temsirolimus and bevacizumab versus interferon alfa and bevacizumab in metastatic renal cell carcinoma: INTORACT trial. J Clin Oncol 2014;32:752-759.

16 Jayson GC, Kerbel R, Ellis LM, Harris AL: Antiangiogenic therapy in oncology: current status and future directions. Lancet 2016;388:518-529.

17 Miles DW, Chan A, Dirix LY, Cortes J, Pivot X, Tomczak P, Delozier T, Sohn JH, Provencher L, Puglisi F, Harbeck N, Steger GG, Schneeweiss A, Wardley AM, Chlistalla A, Romieu G: Phase III study of bevacizumab plus docetaxel compared with placebo plus docetaxel for the first-line treatment of human epidermal growth factor receptor 2-negative metastatic breast cancer. J Clin Oncol 2010;28:3239-3247.

18 Brufsky AM, Hurvitz S, Perez E, Swamy R, Valero V, O’Neill V, Rugo HS: RIBBON-2: a randomized, doubleblind, placebo-controlled, phase III trial evaluating the efficacy and safety of bevacizumab in combination with chemotherapy for second-line treatment of human epidermal growth factor receptor 2-negative metastatic breast cancer. J Clin Oncol 2011;29:4286-4293.
19 Robert NJ, Dieras V, Glaspy J, Brufsky AM, Bondarenko I, Lipatov ON, Perez EA, Yardley DA, Chan SY, Zhou X, Phan SC, O'Shaughnessy J: RIBBON-1: randomized, double-blind, placebo-controlled, phase III trial of chemotherapy with or without bevacizumab for first-line treatment of human epidermal growth factor receptor 2-negative, locally recurrent or metastatic breast cancer. J Clin Oncol 2011;29:1252-1260.

20 Fuchs CS, Tomasek J, Yong CJ, Dumitru F, Passalacqua R, Goswami C, Safran H, Dos Santos LV, Aprile G, Ferry DR, Melichar B, Tehfe M, Topuzov E, Zalcberg JR, Chau I, Campbell W, Sivanandan C, Pikiel J, Koshiji M, Hsu Y, Liepa AM, Gao L, Schwartz JD, Tabernero J: Ramucirumab monotherapy for previously treated advanced gastric or gastro-oesophageal junction adenocarcinoma (REGARD): an international, randomised, multicentre, placebo-controlled, phase 3 trial. Lancet 2014;383:31-39.

21 Wilke H, Muro K, Van Cutsem E, Oh SC, Bodoky G, Shimada Y, Hironaka S, Sugimoto N, Lipatov O, Kim TY, Cunningham D, Rougier P, Komatsu Y, Ajani J, Emig M, Carlesi R, Ferry D, Chandrawansa K, Schwartz JD, Ohtsu A: Ramucirumab plus paclitaxel versus placebo plus paclitaxel in patients with previously treated advanced gastric or gastro-oesophageal junction adenocarcinoma (RAINBOW): a double-blind, randomised phase 3 trial. Lancet Oncol 2014;15:1224-1235.

22 Garon EB, Ciuleanu TE, Arrieta O, Prabhash K, Syrigos KN, Goksel T, Park K, Gorbunova V, Kowalyszyn RD, Pikiel J, Czyzewicz G, Orlov SV, Lewanski CR, Thomas M, Bidoli P, Dakhil S, Gans S, Kim JH, Grigorescu A, Karaseva N, Reck M, Cappuzzo F, Alexandris E, Sashegyi A, Yurasov S, Perol M: Ramucirumab plus docetaxel versus placebo plus docetaxel for second-line treatment of stage IV non-small-cell lung cancer after disease progression on platinum-based therapy (REVEL): a multicentre, double-blind, randomised phase 3 trial. Lancet 2014;384:665-673.

23 Tabernero J, Yoshino T, Cohn AL, Obermannova R, Bodoky G, Garcia-Carbonero R, Ciuleanu TE, Portnoy DC, Van Cutsem E, Grothey A, Prausova J, Garcia-Alfonso P, Yamazaki K, Clingan PR, Lonardi S, Kim TW, Simms L, Chang SC, Nasroulah F: Ramucirumab versus placebo in combination with second-line FOLFIRI in patients with metastatic colorectal carcinoma that progressed during or after first-line therapy with bevacizumab, oxaliplatin, and a fluoropyrimidine (RAISE): a randomised, double-blind, multicentre, phase 3 study. Lancet Oncol 2015;16:499-508.

24 Van Cutsem E, Tabernero J, Lakomy R, Prenen H, Prausova J, Macarulla T, Ruff P, van Hazel GA, Moiseyenko V, Ferry D, McKendrick J, Polikoff J, Tellier A, Castan R, Allegra C: Addition of aflibercept to fluorouracil, leucovorin, and irinotecan improves survival in a phase iii randomized trial in patients with metastatic colorectal cancer previously treated with an oxaliplatinbased regimen. J Clin Oncol 2012;30:3499-3506.

25 Torimura T, Iwamoto H, Nakamura T, Abe M, Ikezono Y, Wada F, Sakaue T, Masuda H, Hashimoto O, Koga H, Ueno T, Yano H: Antiangiogenic and antitumor activities of aflibercept, a soluble VEGF receptor-1 and -2 , in a mouse model of hepatocellular carcinoma. Neoplasia 2016;18:413-424.

26 Motzer RJ, Hutson TE, Cella D, Reeves J, Hawkins R, Guo J, Nathan P, Staehler M, de Souza P, Merchan JR, Boleti E, Fife K, Jin J, Jones R, Uemura H, De Giorgi U, Harmenberg U, Wang J, Sternberg CN, Deen K, McCann L, Hackshaw MD, Crescenzo R, Pandite LN, Choueiri TK: Pazopanib versus sunitinib in metastatic renal-cell carcinoma. N Engl J Med 2013;369:722-731.
27 Van Cutsem E, Bajetta E, Valle J, Kohne CH, Hecht JR, Moore M, Germond C, Berg W, Chen BL, Jalava T, Lebwohl D, Meinhardt G, Laurent D, Lin E: Randomized, placebo-controlled, phase III study of oxaliplatin, fluorouracil, and leucovorin with or without PTK787/ZK 222584 in patients with previously treated metastatic colorectal adenocarcinoma. J Clin Oncol 2011;29:2004-2010.

28 Carrato A, Swieboda-Sadlej A, Staszewska-Skurczynska M, Lim R, Roman L, Shparyk Y, Bondarenko I, Jonker DJ, Sun Y, De la Cruz JA, Williams JA, Korytowsky B, Christensen JG, Lin X, Tursi JM, Lechuga MJ, Van Cutsem E: Fluorouracil, leucovorin, and irinotecan plus either sunitinib or placebo in metastatic colorectal cancer: a randomized, phase III trial. J Clin Oncol 2013;31:1341-1347

29 Grothey A, Van Cutsem E, Sobrero A, Siena S, Falcone A, Ychou M, Humblet Y, Bouche O, Mineur L, Barone C, Adenis A, Tabernero J, Yoshino T, Lenz HJ, Goldberg RM, Sargent DJ, Cihon F, Cupit L, Wagner A, Laurent D: Regorafenib monotherapy for previously treated metastatic colorectal cancer (CORRECT): an international, multicentre, randomised, placebo-controlled, phase 3 trial. Lancet 2013;381:303-312.

30 Bruix J, Qin S, Merle P, Granito A, Huang YH, Bodoky G, Pracht M, Yokosuka O, Rosmorduc O, Breder V, Gerolami R, Masi G, Ross PJ, Song T, Bronowicki JP, Ollivier-Hourmand I, Kudo M, Cheng AL, Llovet JM, Finn RS, LeBerre MA, Baumhauer A, Meinhardt G, Han G: Regorafenib for patients with hepatocellular carcinoma who progressed on sorafenib treatment (RESORCE): a randomised, double-blind, placebocontrolled, phase 3 trial. Lancet 2017;389:56-66.

31 Reck M, Kaiser R, Mellemgaard A, Douillard JY, Orlov S, Krzakowski M, von Pawel J, Gottfried M, Bondarenko I, Liao M, Gann CN, Barrueco J, GaschlerMarkefski B, Novello S: Docetaxel plus nintedanib versus docetaxel plus placebo in patients with previously treated non-small-cell lung cancer (LUME-Lung 1): a phase 3, double-blind, randomised controlled trial. Lancet Oncol 2014;15:143-155.

32 Ledermann JA, Embleton AC, Raja F, Perren TJ, Jayson GC, Rustin GJS, Kaye SB, Hirte H, Eisenhauer E, Vaughan M, Friedlander M, Gonzalez-Martin A, Stark D, Clark E, Farrelly L, Swart AM, Cook A, Kaplan RS, Parmar MKB: Cediranib in patients with relapsed platinum-sensitive ovarian cancer (ICON6): a randomised, double-blind, placebo-controlled phase 3 trial. Lancet 2016;387:1066-1074.

33 Li J, Qin S, Xu J, Xiong J, Wu C, Bai Y, Liu W, Tong J, Liu Y, Xu R, Wang Z, Wang Q, Ouyang X, Yang Y, Ba Y, Liang J, Lin X, Luo D, Zheng R, Wang X, Sun G, Wang L, Zheng L, Guo H, Wu J, Xu N, Yang J, Zhang H, Cheng Y, Wang N, Chen L, Fan Z, Sun P, Yu H: Randomized, double-blind, placebo-controlled phase III trial of apatinib in patients with chemotherapyrefractory advanced or metastatic adenocarcinoma of the stomach or gastroesophageal junction. J Clin Oncol 2016;34:1448-1454.

34 Jain RK, Duda DG, Clark JW, Loeffler JS: Lessons from phase III clinical trials on anti-VEGF therapy for cancer. Nat Clin Pract Oncol 2006;3:24-40.

35 Osada T, Chong G, Tansik R, Hong T, Spector N, Kumar R, Hurwitz HI, Dev I, Nixon AB, Lyerly HK, Clay T, Morse MA: The effect of anti-VEGF therapy on immature myeloid cell and dendritic cells in cancer patients. Cancer Immunol Immunother 2008;57:1115-1124.

36 Baker LC, Boult JK, Thomas M, Koehler A, Nayak T, Tessier J, Ooi CH, Birzele F, Belousov A, Zajac M, Horn C, LeFave C, Robinson SP: Acute tumour response to a bispecific Ang-2-VEGF-A antibody: insights from multiparametric MRI and gene expression profiling. Br J Cancer 2016;115:691-702. 
37 Kloepper J, Riedemann L, Amoozgar Z, Seano G, Susek K, Yu V, Dalvie N, Amelung RL, Datta M, Song JW, Askoxylakis V, Taylor JW, Lu-Emerson C, Batista A, Kirkpatrick ND, Jung K, Snuderl M, Muzikansky A, Stubenrauch KG, Krieter O, Wakimoto H, Xu L, Munn LL, Duda DG, Fukumura D, Batchelor TT, Jain RK: Ang-2/VEGF bispecific antibody reprograms macrophages and resident microglia to anti-tumor phenotype and prolongs glioblastoma survival. Proc Nat Acad Sci U S A 2016;113:4476-4481.

38 Schmittnaegel M, Rigamonti N, Kadioglu E, Cassara A, Wyser Rmili C, Kiialainen A, Kienast Y, Mueller HJ, Ooi CH, Laoui D, De Palma M: Dual angiopoietin-2 and VEGFA inhibition elicits antitumor immunity that is enhanced by PD-1 checkpoint blockade. Sci Transl Med 2017;9.
39 Allen E, Jabouille A, Rivera LB, Lodewijckx I, Missiaen R, Steri V, Feyen K, Tawney J, Hanahan D, Michael IP, Bergers G: Combined antiangiogenic and anti-PD-L1 therapy stimulates tumor immunity through HEV formation. Sci Transl Med 2017;9

40 Tian L, Goldstein A, Wang H, Ching Lo H, Sun Kim I, Welte T, Sheng K, Dobrolecki LE, Zhang X, Putluri N, Phung TL, Mani SA, Stossi F, Sreekumar A, Mancini MA, Decker WK, Zong C, Lewis MT, Zhang XH: Mutual regulation of tumour vessel normalization and immunostimulatory reprogramming. Nature 2017;544: 250-254.
41 Hodi FS, Lawrence D, Lezcano C, Wu X, Zhou J, Sasada T, Zeng W, Giobbie-Hurder A, Atkins MB, Ibrahim N, Friedlander P, Flaherty KT, Murphy GF, Rodig S, Velazquez EF, Mihm MC Jr, Russell S, DiPiro PJ, Yap JT, Ramaiya N, Van den Abbeele AD, Gargano M, McDermott D: Bevacizumab plus ipilimumab in patients with metastatic melanoma. Cancer Immunol Res 2014;2:632-642.

42 El-Kenawi AE, El-Remessy AB: Angiogenesis inhibitors in cancer therapy: mechanistic perspective on classification and treatment rationales. Br J Pharmacol 2013;170:712-729. 